\title{
Thirty Meter Telescope Adaptive Optics System Error Budgets and Requirements Traceability
} Kayla Hardie ${ }^{\mathrm{a}}$, Gelys Trancho ${ }^{\mathrm{a}}$, Lianqi Wang ${ }^{\mathrm{a}}$, Corinne Boyer ${ }^{\mathrm{a}}$, Glen Herriot ${ }^{\mathrm{b}}$, Scott Roberts ${ }^{\mathrm{a}}$, Brent Ellerbroek ${ }^{\mathrm{a}}$, Jean-Pierre Veran ${ }^{\mathrm{b}}$, John Rogers ${ }^{\mathrm{a}}$

${ }^{a}$ Thirty Meter Telescope International Observatory, 100 W. Walnut St., Pasadena, CA, USA 91124;

bational Research Council Canada, 5071 West Saanich Rd., Victoria, BC, Canada V9E 2E7

\begin{abstract}
The Thirty Meter Telescope (TMT) uses error budgets to understand and track the expected science parameters of the Observatory. In this paper, we demonstrate how the top-level requirements for wavefront error in both Multi-Conjugate Adaptive Optics (MCAO) and Natural Guide Star Adaptive Optics (NGSAO) modes have been decomposed and allocated between various sources that may cause performance degradation. We also show how those values have been integrated into the requirements for each individual subsystem. By integrating these error budgets into our requirements management process, we are able to maintain traceability between science and design, and understand how changes at a low-level could affect the overall AO performance of the Thirty Meter Telescope.
\end{abstract}

Keywords: TMT, NFIRAOS, WFE, Budget, Requirements, Traceability

\section{INTRODUCTION}

The TMT NFIRAOS NGSAO and LGS MCAO Wavefront Error Budget ${ }^{1}$ takes the science requirements (SRD) for wavefront error and breaks them down following the TMT system decomposition. Observatory architecture requirements $(\mathrm{OAD})$ are managed by Systems Engineering, and they are further decomposed into lower-level requirements which are managed by each subsystem team. The budget is split into LGS MCAO mode (Table 1) and NGSAO mode (Table 2).

To reduce the number of requirements and facilitate future changes, a single requirement is used for subsystems that have the same WFE allocation regardless of whether LGS MCAO (on-axis) or NGSAO mode. These allocations are shown in gray in the NGSAO budget, which, if also gray in the LGS on-axis budget, also denotes commonality between the LGS MCAO on-axis, 17"x17", and 30" field of view allocations. Some requirements in Table 1 are shown in pink, denoting common terms that, if changed, would require an update to the telescope seeing-limited budget. Values shown in white are for any allocations specific to LGS MCAO mode.

\subsection{LGS MCAO Budget}

The LGS MCAO budget (Table 1) is split into High Order and Low Order modes, with each showing the requirements decomposition based on on-axis, 17"x17", or 30" (nm) field of views. The higher order modes contain all modes beyond tip, tilt, plate scale, and global focus. Low order modes include global tip/tilt, focus, and plate scale that are controlled only by low order NGS WFS.

\subsection{NGSAO Budget}

The NGSAO budget (Table 2) is split into High Order and Low Order modes, and the requirements are decomposed based on $\mathrm{mR}=8$ or $\mathrm{mR}=12$ guide stars. The low order modes include tip/tilt only. 
Table 1. TMT NFIRAOS LGS MCAO Wavefront Error Budget.

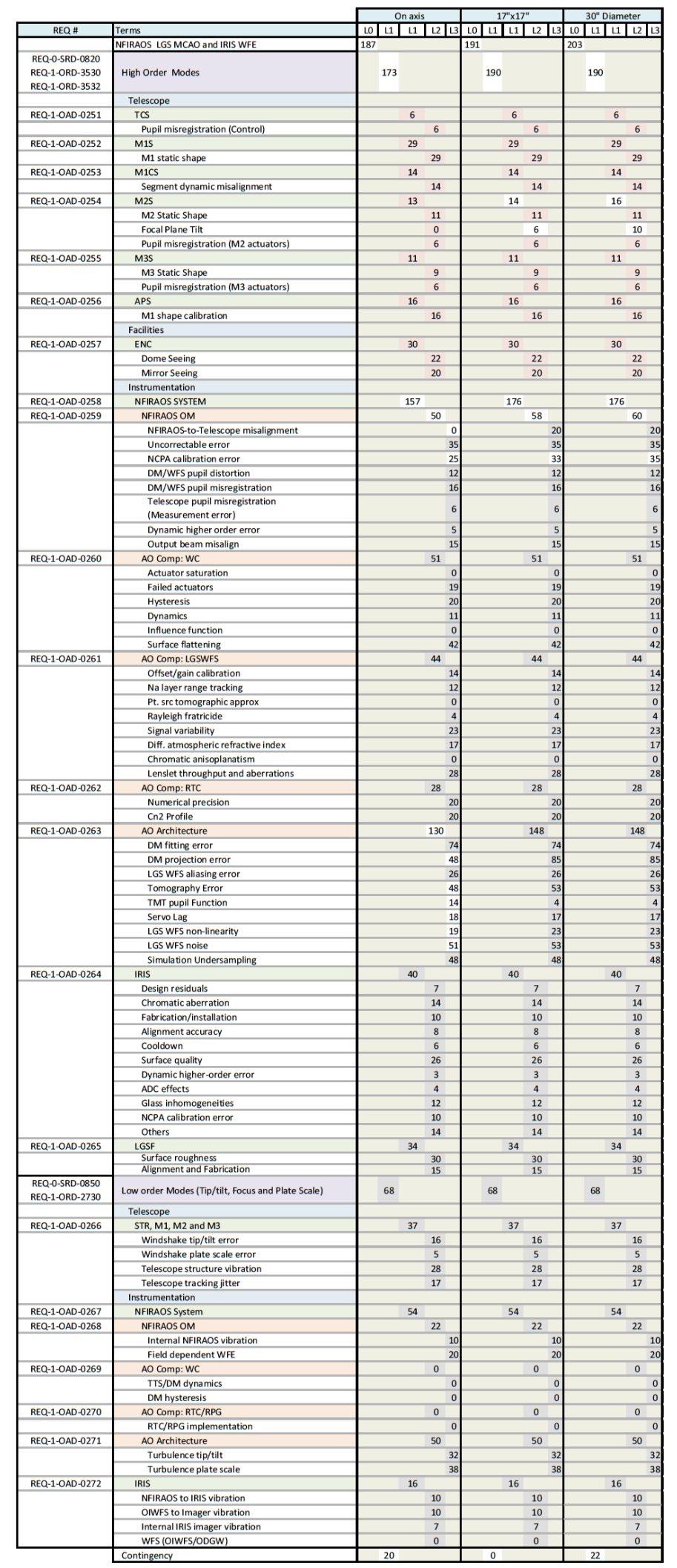


Table 2. TMT NFIRAOS NGSAO Wavefront Error Budget.

\begin{tabular}{|c|c|c|c|}
\hline & & & $m R=8$ \\
\hline REQ\# & Terms & LO & \begin{tabular}{l|l}
$\mathrm{L} 1$ & $\mathrm{~L}$ \\
\end{tabular} \\
\hline $\begin{array}{l}\text { REQ-0-SRD-880 } \\
\text { REQ-0-SRD-881 }\end{array}$ & NFIRAOS NGSAO and IRIS WFE & 15 & \\
\hline $\begin{array}{l}\text { REQ-1-ORD-3670 } \\
\text { REQ-1-ORD-3671 }\end{array}$ & High Order Modes & & 149 \\
\hline & Telescope & & \\
\hline REQ-1-OAD-0251 & TCS & & \\
\hline & Pupil misregistration (Control) & & \\
\hline REQ-1-OAD-0252 & M1S & & \\
\hline & M1 static shape & & \\
\hline REQ-1-OAD-0253 & M1CS & & \\
\hline & Segment dynamic misalignment & & \\
\hline REQ-1-OAD-0254 & M2S & & \\
\hline & M2 Static Shape & & \\
\hline & Focal Plane Tilt & & \\
\hline & Pupil misregistration (M2 actuators) & & \\
\hline REQ-1-OAD-0255 & M3S & & \\
\hline & M3 Static Shape & & \\
\hline & Pupil misregistration (M3 actuators) & & \\
\hline REQ-1-OAD-0256 & APS & & \\
\hline & M1 shape calibration & & \\
\hline & Facilities & & \\
\hline REQ-1-OAD-0257 & ENC & & \\
\hline & Dome Seeing & & \\
\hline & Mirror Seeing & & \\
\hline & Instrumentation & & \\
\hline REQ-1-OAD-0273 & NFIRAOS SYSTEM & & \\
\hline REQ-1-OAD-0274 & NFIRAOSOM & & \\
\hline & NFIRAOS-to-Telescope misalignment & & \\
\hline & Uncorrectable error & & \\
\hline & NCPA calibration error & & \\
\hline & Registration Drifts after Calibration & & \\
\hline & Image Quality at Pyramid tip & & \\
\hline & $\begin{array}{l}\text { Telescope pupil misregistration } \\
\text { (Measurement error) }\end{array}$ & & \\
\hline & Dynamic higher order error & & \\
\hline & Output beam misalign & & \\
\hline REQ-1-OAD-0260 & AO Comp: WC & & \\
\hline & Actuator saturation & & \\
\hline & Falled actuators & & \\
\hline & Hysteresis & & \\
\hline & Dynamics & & \\
\hline & Influence function & & \\
\hline & Surface flattening & & \\
\hline REQ-1-OAD-0275 & AO Comp: PWFS WFS & & \\
\hline & Optical gain tracking & & \\
\hline & Pupil image location & & \\
\hline & Imperfect pyramid & & \\
\hline & Pupil image quality & & \\
\hline & CCD charge diffusion & & \\
\hline & Pupil image distortion & & \\
\hline & Modulation errors & & \\
\hline REQ-1-OAD-0276 & AO Comp: RTC & & \\
\hline & Numerical precision & & \\
\hline REQ-1-OAD-0277 & AO Architecture & & \\
\hline & DM fitting error & & \\
\hline & PWFS aliasing error & & \\
\hline & TMT pupil Function & & \\
\hline & Servo Lag & & \\
\hline 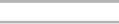 & WFS non-linearity & & \\
\hline & WFS noise & & \\
\hline & Simulation Undersampling & & \\
\hline REQ-1-OAD-0264 & IRIS & & \\
\hline & Design residuals & & \\
\hline & Chromatic aberration & & \\
\hline & Fabrication/installation & & \\
\hline & Alignment accuracy & & \\
\hline & Cooldown & & \\
\hline & Surface quality & & \\
\hline & Dynamic higher-order error & & \\
\hline & ADC effects & & \\
\hline & Glass inhomogeneities & & \\
\hline & NCPA calibration error & & \\
\hline & Others & & \\
\hline REQ-1-ORD-3669 & Low Order Modes (Tip/Tilt and Focus) & & 29 \\
\hline & Telescope & & \\
\hline REQ-1-OAD-0278 & $\mathrm{STR}, \mathrm{M} 1, \mathrm{M} 2$ and $\mathrm{M} 3$ & & \\
\hline & Windshake tip/tilt error & & \\
\hline & Telescope structure vibration & & \\
\hline & Telescope tracking jitter & & \\
\hline & Instrumentation & & \\
\hline REQ-1-OAD-0279 & NFIRAOS System & & \\
\hline REQ-1-OAD-0280 & NFIRAOSOM & & \\
\hline & Internal NFIRAOS vibration & & \\
\hline REQ-1-OAD-0281 & AO Comp: WC & & \\
\hline & $\pi S / D M$ dynamics & & \\
\hline & DM hysteresis & & \\
\hline REQ-1-OAD-0282 & AO Comp: RTC/RPG & & \\
\hline & RTC/RPG implementation & & \\
\hline REQ-1-OAD-0283 & AO Architecture & & \\
\hline & Turbulence tip/tilt & & \\
\hline REQ-1-OAD-0272 & IRIS & & \\
\hline & NFIRAOS to IRIS vibration & & \\
\hline & OIWFS to Image r vibration & & \\
\hline & Internal IRIS imager vibration & & \\
\hline & WFS (OIWFS/ODGW) & & \\
\hline & Contingency & & 45 \\
\hline
\end{tabular}




\section{TRACEABILITY TOOLS}

TMT Error Budgets are generated in Excel and then integrated into the Rational Dynamic Object Oriented Requirements System (DOORS) ${ }^{3}$. DOORS is the source for all TMT requirements as the database allows us to link parent/child requirements together while also being able to generate user-friendly requirement documents for each subsystem.

Each top-level wavefront error allocation from the budget is translated into an individual requirement in DOORS. These allocations then flow down to a lower-level requirement for each contributing subsystem. Links are created between the requirements, providing us with full traceability from top to bottom. Traceability reports are generated directly from DOORS or using the DOORS Trace Tree ${ }^{4}$ tool which shows us the same information in a graphical output (Figure 1, Figure 2). The graphical outputs are extremely useful when trying to identify if a requirement has not yet been traced, or has been mis-traced, to parent or child requirements.

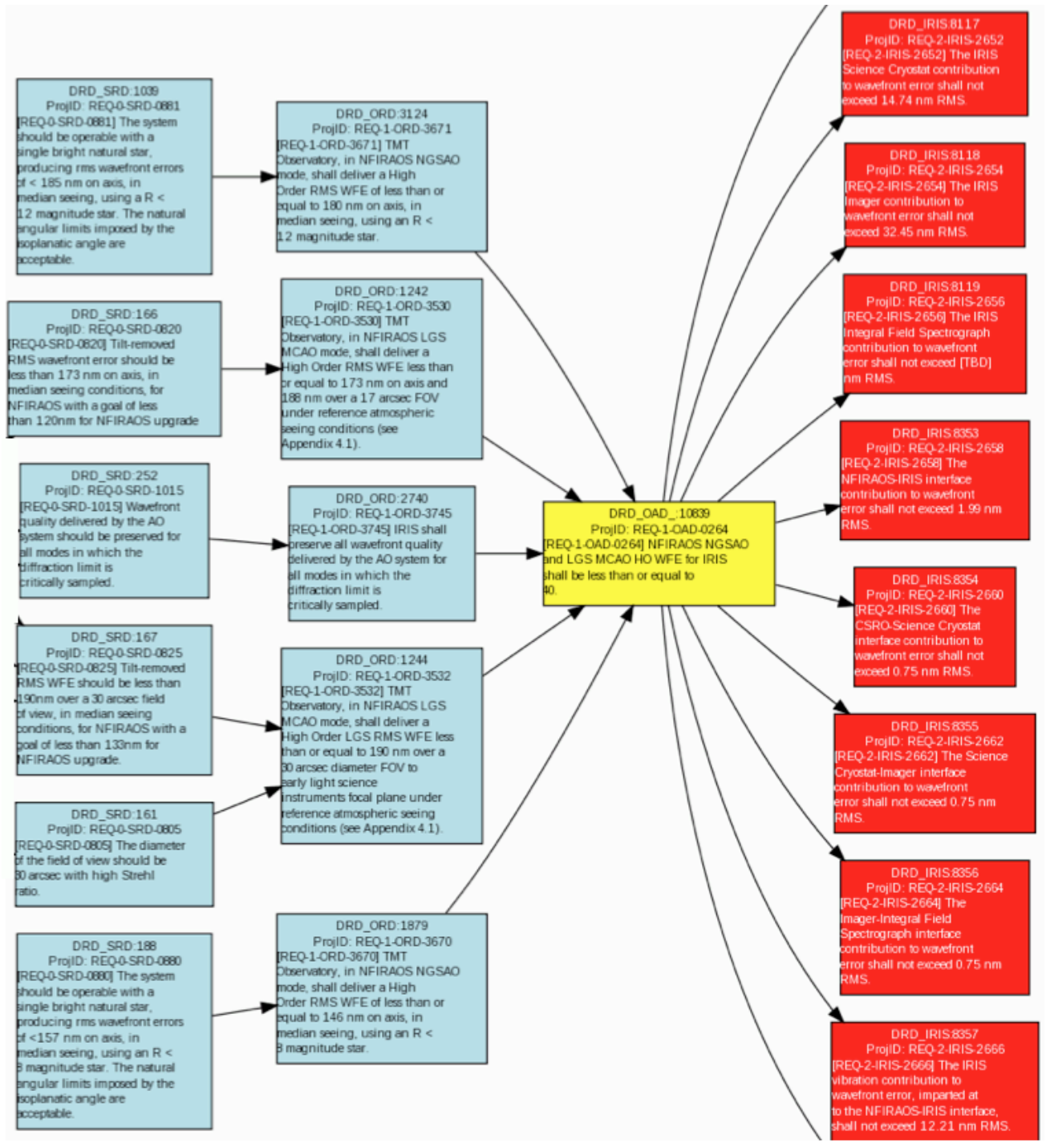

Figure 1. NGSAO \& LGS MCAO WFE Requirement Traceability for IRIS (note: not all IRIS requirements shown). 


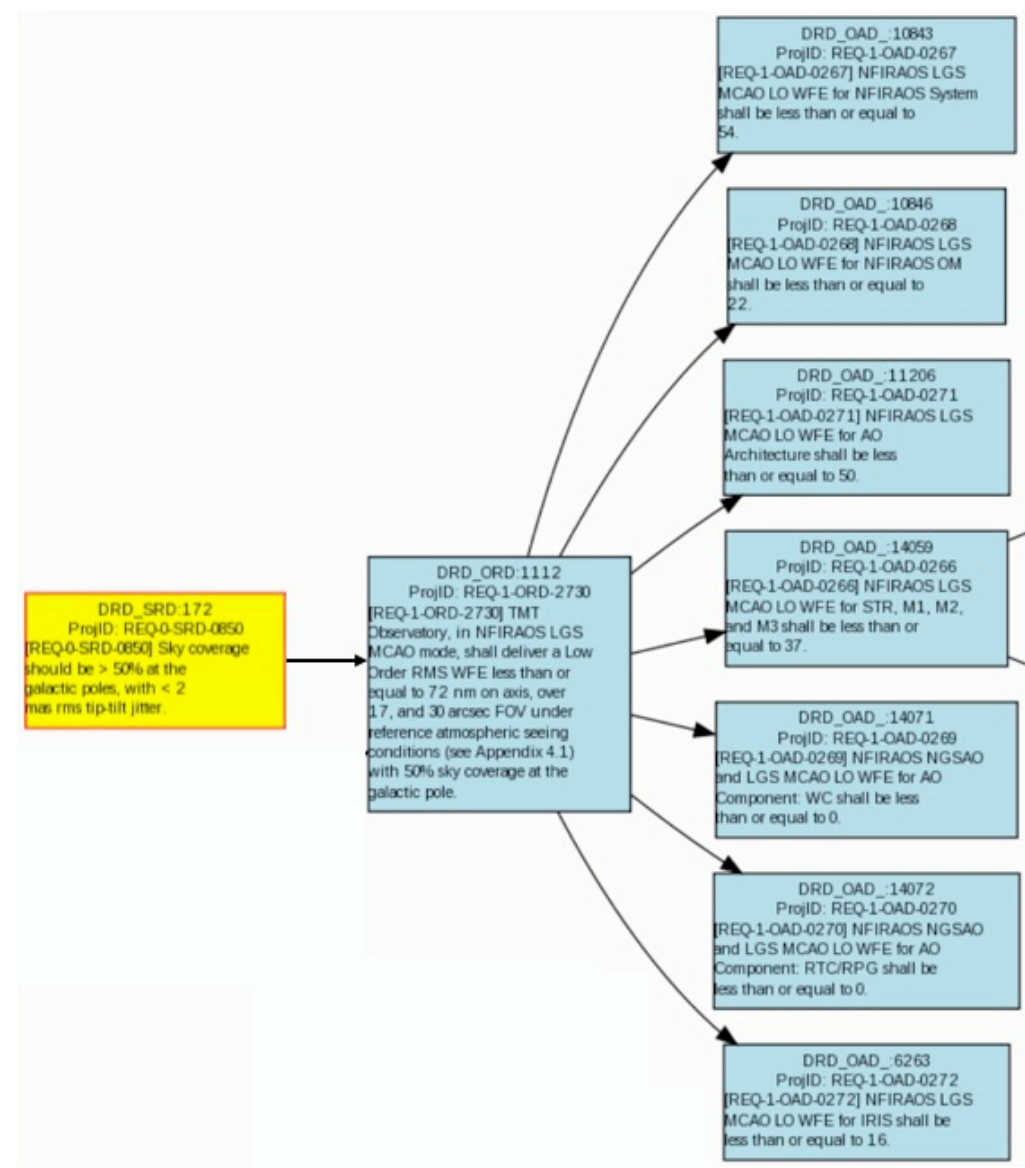

Figure 2. LGS MCAO Requirement Flowdown Example from Trace Tree.

\section{NEXT STEPS}

Work on the TMT NFIRAOS NGSAO and LGS MCAO Wavefront Error Budget is still in progress. Future work will include activities such as evolving the IRIS imager 34"x34" field of view allocations and incorporating point source sensitivity.

\section{SUMMARY}

Error budgets are an essential Systems Engineering tool to estimate the TMT AO system's future performance. Systems Engineering, with input from subsystem teams, is responsible for tracking changes to the overall $\mathrm{AO}$ error budget. By using the error budgets in conjunction with our traceability tools, we are able to quickly assess how changes in one part of the system may impact higher-level requirements, helping us to minimize risks associated with meeting system and science requirements. 


\section{REFERENCES}

[1] TMT Observatory NFIRAOS LGS MCAO, NGSAO and IRIS Imager Wavefront Error Budget and Current Best Estimate, TMT.AOS.COR.16.062

[2] TMT Observatory NFIRAOS LGS MCAO, NGSAO and IRIS Imager Wavefront Error Budget and Current Best Estimate Description, TMT.AOS.TEC.08.015

[3] IBM Dynamic Object Oriented Requirements System, http://www03.ibm.com/software/products/en/ratidoor

[4] DOORS Trace Tree, https://trace-tree.tmt.org/ 